\title{
Full-wave Analysis of Periodic Baffle System in Beamforming Applications
}

\author{
Yuriy TASINKEVYCH, Eugeniusz DANICKI \\ Institute of Fundamental Technological Research \\ Polish Academy of Sciences \\ Pawińskiego 5B, 02-106 Warszawa, Poland \\ e-mail: yurijtas@ippt.gov.pl \\ (received July 13, 2010; accepted September 6, 2010)
}

\begin{abstract}
Mixed boundary-value problem for periodic baffles in acoustic medium is solved with help of the method developed earlier in electrostatics. The nice feature of the method is that the resulting matrices are relatively easy for computations and that the results satisfy exactly the energy conservation law. Illustrative numerical examples present the wave-beam steering (in the far-field) in a baffle system that may be considered as a model of one-dimensional ultrasonic transducer array.
\end{abstract}

Keywords: sound field calculation, linear phased arrays, BIS expansion, far-field radiation pattern, beam-steering, transducer arrays, composite transducers, scattering, generation, fields.

\section{Introduction}

A typical one-dimensional ultrasonic array transducer consists of alternate sets of acoustically different materials: piezoelectric, which responds to the incident waves by electric signal, and acoustically isolating material (like epoxy) between them (SEO, YEN, 2007; Yen et al., 2000). By controlling the phase and amplitude of the excitation signals of different elements the beam generated by the array can be dynamically steered in the region of interest and focused at a given point. The phased arrays are widely and successfully used in medical ultrasonic diagnostics nowadays (Thomenius, 1996; Trots et al., 2008; 2009; ZEMP et al., 2003). Their properties, including flexible control and signal processing, high inspection speed and fast imaging capabilities, give rise to their advantages over conventional ultrasonic transducers. The nondestructive evaluation and testing is another area where the ultrasonic phased arrays has been receiving great attention recently (DRINKWATER, WilCOX, 2006; KRAMB, 2007). 
There are different approaches to the linear phased array modeling described in the literature. The beam profile modeling is based on the intuitive representation of an array as a set of simple point sources (AHMAD et al., 2005; WoOH, SHI, 1998). In the point spread function modeling (CHIAO, Thomas, 1994; LingVALL et al., 2003) the ability of a phased array to visualize a point reflector (by means of certain imaging algorithm) is modeled. For this purpose the ultrasonic data from the array due to a point reflector at a particular spatial position are simulated first. Then the image of the reflector is plotted using the appropriate imaging algorithm applied to the simulated data.

Both these methods must apply certain model of the individual array element (they are typically piezoelectric beams separated by epoxy layers). There are different methods of modeling the array elements, including finite element analysis (LERCH, 1990; YARALIOGLU et al., 2005) or Huygens principle (MCNAB et al., 1990; WoOH, SHI, 1999). In the later case, usually the integration of a series of point or line sources is performed to obtain the element directivity function due to the finite size of the array element. The above approaches to the modeling of array transducer assume that the individual elements respond to the incident wave pressure independent on each other yielding the electric signal proportional to the incident wave amplitude.

However, since piezoelectric materials are closer to hard, and epoxy is closer to soft acoustic materials, the Bragg scattering occurs when the incident wave scatters from the array. This phenomenon must somehow distort the local acoustic pressure on piezoelectric elements of the array affecting its electric response. In this paper an approach for modelling the ultrasonic linear array transducer is proposed, which is based on the rigorous full-wave analysis of the corresponding boundary-value problem for wave excitation or scattering. The considered system consists of periodic acoustically hard strips (baffles) where the normal acoustic vibration vanishes (ERBAS, ABrahams, 2007), and between them are acoustically soft domains where the acoustic pressure vanishes (or it is given constant in the excitation problem). This is a mixed (Dirichlet-Neumann) boundary-value problem that we deal with: the given pressure between baffles models the wavebeam generation, and the pressure exerted by the incident and scattered waves on the acoustically hard baffles models the response signal from the individual piezoelectric element of the array transducer. A similar system of baffles modeling the phased array transducer was considered for example in (KUHNICKE, 2007). An efficient method developed in electrostatics (DANICKI, 2004) is found suitable for rigorous solution of the above-mentioned problem for planar periodic baffles. Similar electrostatic method can be applied for analyzing finite (aperiodic) system of baffles, as well (BoERsma, DANICKI, 1993; DANICKI, 1996; TASINKEVYCH, 2004). Moreover, the method results in the small system of equations to be solved numerically, and yields the results which satisfy exactly the energy conservation law (with machine accuracy (DANICKI, 1999; 2002)), in both the generation and scattering cases. 


\section{The boundary-value problems for periodic strips}

\subsection{Surface harmonic impedance of acoustic half-space}

Two-dimensional harmonic wave-field $e^{j(\omega t-\xi x-\eta z)}$ is considered independent of $y$ in the acoustic media governed by equations for acoustic potential $\varphi$, pressure $p$ and particle velocity $\mathbf{v}(t$ - time, $x, y, z$ - spatial coordinates oriented as in Fig. 1, $\omega, \xi, \eta$-angular temporal and spatial frequencies):

$$
\begin{aligned}
\nabla^{2} \varphi+k^{2} \varphi & =0, \\
\mathbf{v}=-\nabla \varphi, \quad p & =j \omega \rho_{a} \varphi,
\end{aligned}
$$

where $k=\omega / c$. Standard notations are applied: $c$ is the sound velocity and $\rho_{a}$ is mass density of the media.

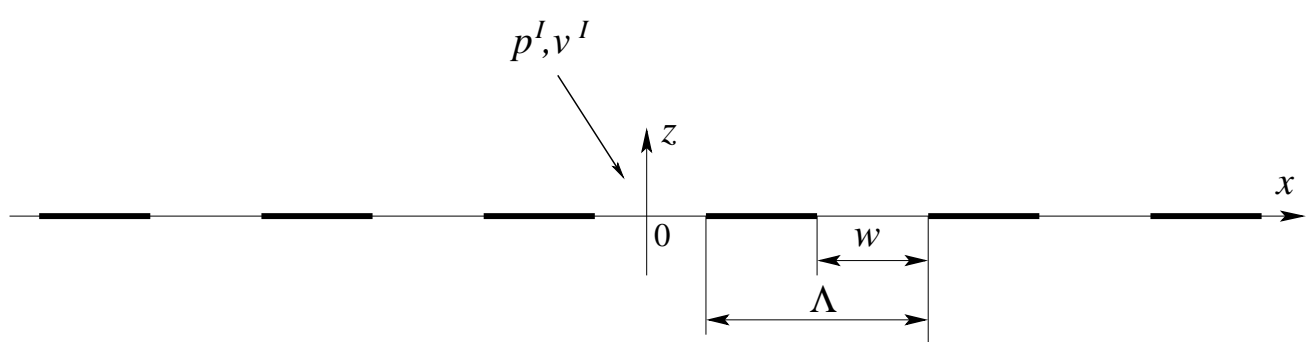

Fig. 1. Periodic system of rigid baffles.

We pay special attention to the wave-field at the plane $z=0$. Assuming known pressure there of the form $p e^{-j \xi x}$ where $p$ is its amplitude, the resulting $z$-component of the particle velocity $\left.v_{z}\right|_{z=0+}$ on the upper side of this plane (denoted by $v$ to shorten notations), can be easily evaluated. Thus, neglecting the exponential term $\exp (j \omega t-j \xi x)$ we obtain:

$$
\begin{gathered}
v=v_{z}=-\varphi_{, z}=\eta /\left(\omega \rho_{a}\right) p=G p, \quad G(\xi)=\eta /\left(\omega \rho_{a}\right), \\
\eta=\sqrt{k^{2}-\xi^{2}}=-j \sqrt{\xi^{2}-k^{2}},
\end{gathered}
$$

where $G$ is the surface harmonic admittance of acoustic half-space. In the above, the relationship between $\varphi$ and $p$ from Eq. (1) has been used. Note, in Eq. (2) $\eta$ is chosen in order to satisfy the radiation condition of the acoustic field at $z \rightarrow \infty$. In the presented method of analysis, we will need the $x$-derivative of pressure $p(x)$ at $z=0$, which is in somewhat symbolic notation $q=p_{, x}=-j \xi p$. Substituting $q$ into Eq. (2) we obtain in spectral domain:

$$
\begin{aligned}
v & =(j G / \xi) p_{, x}=g(\xi) q, \\
g(\xi) & =\frac{j}{\omega \rho_{a}} \frac{\eta}{\xi} .
\end{aligned}
$$


The function $g(\xi)$ will replace the Eq. (1) in all the analysis which follows for the pressure and velocity on the plane $z=0+$. Note that for large values of its argument the following asymptotic equality holds:

$$
g(\xi \rightarrow \pm \infty)=g_{\infty} S_{\xi},
$$

where $g_{\infty}=1 /\left(\omega \rho_{a}\right)$ is the acoustic admittance divided by $k$, and $S_{\xi}=1$ for $\xi \geq 0$ and -1 otherwise, for arbitrary real $\xi$. Inside the media, the acoustic potential $\varphi$ generated by the given pressure distribution $p$ at $z=0$ is

$$
\varphi(x, z)=-j \frac{p}{\omega \rho_{a}} e^{-j \xi x-j \eta z} .
$$

\subsection{Boundary conditions at the baffle plane}

\subsubsection{Sound generation - given pressure between baffles}

A periodic system of the acoustically hard baffles distributed along $x$-axis with period $\Lambda$ on the boundary $z=0$ of the acoustic medium spanning for $z>0$ is considered (see Fig. 1). The $z$-component of particle velocity on baffles vanishes, and in the slot between baffles, a harmonic pressure of amplitude $p_{l}$ (constant over an entire slot) excites the wave-field in the medium; $l \Lambda$ describes the position of the given $l$-th slot center along $x$-axis.

For $\Lambda-w$ wide baffles centered at $=l \Lambda+\Lambda / 2(l$ - any integer $)$ the boundary conditions at $z=0$ are:

$$
\begin{aligned}
q & =0, \quad x \in(-w / 2, w / 2)+l \Lambda \text { between strips, } \\
v & =0, \quad x \notin(-w / 2, w / 2)+l \Lambda \text { on strips, } \\
p(l \Lambda) & =p_{l}, \quad \text { in the middle between strips, }
\end{aligned}
$$

where $p_{l}$ are given values. They are constant in given slots between baffles due to the condition $q=0$ there. The solutions to the boundary-value problem of interest are functions $p(x)$ and $v(x)$ at $z=0$ plane, which will be searched in the form of Bloch series, natural for the considered periodic system. The field inside the medium, $z>0$, can be evaluated from Eq. (5).

\subsubsection{Sound detection - the scattering by baffles}

The plane incident wave of the form $e^{j\left(\omega t-\xi_{I} x+\eta_{I} z\right)}$ is assumed, yielding the following wave-field at $z=0:\left(p^{I}, v^{I}\right) e^{-j \xi_{I} x}$, where $p^{I}$ and $v^{I}$ are the pressure and velocity (z-component) amplitudes of the incident field, respectively. It is convenient for the further analysis to rewrite the wavenumber component $\xi_{I}$ as follows: $\xi_{I}=r+I K$, where $K=2 \pi / \Lambda$ is spatial wavenumber of the baffle array, $r \in(0, K)$ is a reduced wavenumber from the first Brillouin zone, $I$ is an arbitrary integer. This yields at $z=0: v^{I}(r) e^{-j(r+I K) x}, p^{I}(r) e^{-j(r+I K) x}$. The relation between $p^{I}$ and $v^{I}$ involves the harmonic admittance $-G$, Eq. (2), because 
the incident wave, although propagating in the upper half-space of the medium, satisfies the radiation condition at $z \rightarrow-\infty$ :

$$
\begin{aligned}
& v^{I}=-G(r+I K) p^{I}, \\
& v^{I}=-g(r+I K) q^{I}, \\
& q^{I}=-j(r+I K) p^{I} .
\end{aligned}
$$

In the case of any non-planar incident wave, its spatial Fourier expansion on the plane $z=0$ should be applied instead, resulting in certain function $v^{I}(r)$; the whole domain of $r \in(0, K)$, as well as numerous values of $I$ must be included in the analysis of such non-plane incident wave. In the presented analysis however, and for the sake of presentation simplicity, we consider only the plane incident wave where $r$ depends on the angle of incidence.

The boundary conditions for full acoustic wave-field are the same as in the previous case, Eqs. (6), except that $p_{l}=0$ is applied. The total force on a baffle exerted by the incident and scattered waves, models the response of the piezoelectric transducer element to the incident acoustic wave.

\section{Application of electrostatic methods}

In periodic systems with period $\Lambda=2 \pi / K$, the wave-field can be represented by the Bloch series like

$$
p(x)=\sum_{n=-\infty}^{\infty} p_{n} e^{-j(r+n K) x}, \quad r \in(0, K)
$$

(for example of acoustic pressure $p$ ) where $r$ is an arbitrary wave-number constrained to one Brillouin zone for the sake of uniqueness of the representation. In the already developed electrostatic techniques, the Bloch components $p_{n}$ are expanded again using the method introduced by Blotekjœr, Ingebrigtsen, and Skeie (BloteKJa et al., 1973), referred to as the BIS expansion method, into the finite series of Legendre polynomials $P_{k}(\cdot)=P_{k}^{(0)}(\cdot)$, which expansion, possessing crucial property to the considered boundary-value problem (ERDELYI et al., 1953):

$$
\begin{gathered}
\sum_{n=-\infty}^{\infty} S_{n} P_{n}(\cos \Delta) e^{-j n K x}=0 \text { for }|x|<w / 2, \\
\sum_{n=-\infty}^{\infty} P_{n}(\cos \Delta) e^{-j n K x}=0 \text { for }|x|>w / 2,
\end{gathered}
$$

where $\Delta=\pi w / \Lambda$, will help us to satisfy the boundary conditions (the above functions are periodic). For $(\Lambda-w)$-wide strips centered at $x=l \Lambda+\Lambda / 2$, 
the corresponding field expansion at $z=0$ applied in the solution to both the boundary-value problems formulated above will be:

$$
\begin{aligned}
q & =\sum_{n=-\infty}^{\infty} q_{n} e^{-j(r+n K) x}, \\
q_{n} & =\sum_{m} \alpha_{m} S_{n-m} P_{n-m}(\cos \Delta), \\
v & =\sum_{n=-\infty}^{\infty} v_{n} e^{-j(r+n K) x}, \\
v_{n} & =\sum_{m} \beta_{m} P_{n-m}(\cos \Delta) .
\end{aligned}
$$

The boundary conditions (6) are fulfilled on the strength of Eqs. (9). Now, we need only to check if the applied wave-field solution satisfies the wave equation inside the media, which equation is represented at the plane $z=0$ by the harmonic admittance $G(r+n K)$ or more convenient, by its version $g$, Eq. (3).

Only this part of the wave-field $(q, v)$ which satisfies radiation condition at $z \rightarrow \infty$ are involved in Eq. (3). Hence, if it is to be applied here, the incident wave-field must be subtracted from the field at $z=0$. This yields the relation for the $n$th Bloch component having wave-number $r+n K\left(\delta_{i j}\right.$ is the Kronecker delta):

$$
\left(v_{n}-v^{I} \delta_{n I}\right)=g(r+n K)\left(q_{n}-q^{I} \delta_{n I}\right), \quad r \in(0, K),
$$

which must be satisfied for all $n$, particularly for large $|n| \gg|I|$ where

$$
g(r \pm N K)= \pm g_{\infty}
$$

(formally, $N=\infty$, but in the applied approximation, $N$ is assumed large but finite - this is the only approximation applied in the method). This is only possible if (BlotekJa et al., 1973):

$$
\beta_{m}=g_{\infty} \alpha_{m}
$$

which substituted into the earlier equation and accounting for the last of Eqs. (7) yields:

$$
\sum_{m} \alpha_{m}\left[g(r+n K) S_{n-m}-g_{\infty}\right] P_{n-m}(\cos \Delta)=2 g(r+n K) q^{I} \delta_{n I} .
$$

Under condition (12), the equations for $\alpha_{m}$ outside the limits $n \in[-N, N]$ and $m \in[-N, N+1]$ (moreover it must be $N>|I|)$ are satisfied automatically, what can be checked by inspection (BlotekJA et al., 1973). Hence, Eq. (14) yields the system of $2 N+1$ equations for $2(N+1)$ unknowns.

It can be now explained what is the source of advantage of the presented method over other methods applied in scattering theory for similar problems. 
The boundary conditions are satisfied exactly by the proposed solution, Eqs. (10), but certain departure is made concerning the equation of motion which is represented here by the harmonic admittance $G$ or $g$, Eqs. (2)-(3). This departure relies on the approximation that $g(r+n K) \equiv g_{\infty}$ for $r+n K$ greater that certain large but finite value (far above the domain of existence of propagating modes in the media). In fact, $g(x) \rightarrow g_{\infty}$ like $1 / x^{2}$ and indeed this approximation can be applied in general, but this is the approximation, the only one in the analysis. In the consequence of it, the wave-field on the baffle plane is represented in finite Fourier series multiplied by the square-root singular function at the baffle edges (BlotekJA et al., 1973). The larger the BIS expansions are, Eqs. (10), that is the larger the applied $N$ is, the better approximation is achieved.

In fact usually, for $K \approx k, N$ can be chosen quite small. The resulting system of equations (14) appended by the one resulting from the last boundary conditions in Eqs. (6), can be easily solved. This last equation can be evaluated by integration of $q=p_{, x}$, Eq. (10), using the Dougall identity (ERDELY et al., 1953):

$$
P_{-\nu}(-\cos \Delta)=-\frac{\sin \nu \pi}{\pi} \sum_{n=-\infty}^{\infty} \frac{S_{n} P_{n}(\cos \Delta)}{\nu-n}
$$

(in somewhat transformed form, using identities $P_{n}(-x)=(-1)^{n} P_{n}(x)$ and $\left.P_{-n-1}=P_{n}\right)$, yielding (DANICKI, 2004):

$$
p_{r}(l \Lambda)=j \sum_{n} \frac{q_{n} e^{-j r l \Lambda}}{r+n K}=-j \sum_{m} \frac{\pi(-1)^{m} \alpha_{m} e^{-j r l \Lambda}}{K \sin \pi r / K} P_{-r / K-m}(-\cos \Delta),
$$

which, being constant between neighboring baffles, is evaluated at $x=l \Lambda$; note the dependence on $r$ indicating its spectral-domain origin.

The solution we seek, must satisfy the condition that the pressure at different slots between baffles takes different (given) values $p_{l}$, dependent on $l$. This requires integration of the above equation over $r \in(0, K)$, which is the inverse Fourier transform of discrete function $p_{l}=p_{r}(l \Lambda)$ defined by (note that the harmonic term $\exp (-j r l \Lambda)$ is already included in the $p_{r}$ evaluated above):

$$
\frac{1}{K} \int_{0}^{K} p_{r}(l \Lambda) \mathrm{d} r=p_{l} .
$$

This finally yields the last condition for $\alpha_{m}$ dependent on $r$ ( $p_{l}$ are given):

$$
\sum_{m}(-1)^{m} \alpha_{m} P_{-r / K-m}(-\cos \Delta)=j \frac{K}{\pi} p_{l} e^{j r l \Lambda} \sin \pi r / K
$$

simple substitution into Eq. (16) verifies that Eq. (17) is satisfied. This is the last equation that must be appended to the system (14) in order to obtain equal number of equations and unknowns. Now, we can evaluate $\alpha_{m}$ dependent on given $p^{I}$ or $p_{l}$, and finally, we can evaluate the wave-field at $z=0$ and elsewhere in the media $z>0$ using expansion (10) and (5). 


\section{Numerical examples}

\subsection{Sound beam-forming by chosen pressure between baffles}

In contrast to the earlier papers (CROMBie et al., 1997; JENSEN, SvEndSEn, 1992; SELfridge et al., 1980), the applied method of analysis yields spatial spectrum of the acoustic pressure at the baffle plane. Taking the advantage of this, the radiation pattern can be simply evaluated from the inverse Fourier transform (as already defined in Eq. (17) on the baffle plane) of $p(\xi)$, dependent on the wave-number $\xi=r+n K$, Eq. (1). The unified representation $p(\xi)$ includes all Bloch orders and is defined by ( $n$ is the integer part of $\xi: n=E(\xi)$; note that $E(-1.5)=-2)$ :

$$
p(\xi)=\frac{q_{n}(\xi-n K)}{-j \xi}, \quad n=E(\xi) .
$$

At the level $z$ above the baffle plane $z=0$, the acoustic pressure behaves according to Eq. (5), thus introducing spatial angular variables: $x=R \sin \theta, z=R \cos \theta$, and $\eta$ given in Eq. (2), the pressure in angular coordinates is:

$$
p_{R}(\theta)=\int_{-\infty}^{\infty} p(\xi) e^{-j R \xi \sin \theta} e^{-j R \eta \cos \theta} \mathrm{d} \xi / K .
$$

At large distance $R \rightarrow \infty$, we may drop this part of the integral representing the localized field at the baffle plane, which depends on imaginary valued $\eta$, Eq. (2). This is made by constraining $\vartheta$ to the domain $(-\pi / 2, \pi / 2)$ in the transformed integration where $\xi=k \sin \vartheta, \eta=k \cos \vartheta$ :

$$
p_{R}(\theta)=\int_{-\pi / 2}^{\pi / 2}(k / K) p(k \sin \vartheta) \cos \vartheta e^{-j k R \cos (\vartheta-\theta)} \mathrm{d} \vartheta,
$$

which integral can be easily evaluated by the stationary phase method (FEDORYUK, 1997; FELSEN, MARCUVITZ, 1973) (the stationary point of interest here is $\theta=\vartheta$ ):

$$
p_{R}(\theta)=p(k \sin \theta) \cos \theta \frac{k}{K} \sqrt{\frac{j 2 \pi}{k R}} e^{-j R k} .
$$

This is an alternate method of evaluation of angular radiation characteristics to the one presented in (Crombie et al., 1997; Jensen, Svendsen, 1992; SelfRIDGE et al., 1980). It can be however, evaluated in more efficient method explained below.

Solving Eqs. (11)-(18), we obtain $n$th Bloch order of the acoustic pressure, $p_{n}=j q_{n} /(r+n K)$ for given $r$, Eq. (10). This is the wave pressure radiated into the half-space $z>0$ at the direction described by the corresponding wavenumbers $\left(\xi_{n}=r+n K, \eta_{n}=\sqrt{k^{2}-(r+n K)^{2}}\right)$ provided that $\eta_{n}$ is real. 
Hence, only limited values of $n$ contribute to the angular radiation pattern at $\theta=\arctan \left(\xi_{n} / \eta_{n}\right)$ (there are multiple directions for small $K$ ). Repeating this for all allowed $r \in(0, K)$ in Eq. (8) (followed by evaluation of $\alpha_{m}$ from Eq. (7)(18) and $q_{n}$ from Eq. (10) in the manner presented above), the angular radiation pattern can be fully reconstructed.

The angular radiation pattern concerns the acoustic amplitude $a$ which is related to the acoustic power by $\Pi=|a|^{2} / 2$. It is easier to evaluate first the normal component of the acoustic Poynting vector $\Pi_{z}$ using the solutions for acoustic pressure $p$ and normal velocity $v$ at $z=0$. For given $r$ and Bloch order $n^{\prime}$ for which $\eta$ is real, it is

$$
\Pi_{z}^{\left(n^{\prime}\right)}=\operatorname{Re}\left\{v_{n} p_{n}^{*}\right\} / 2, \quad \Pi_{z}=\sum_{n^{\prime}} \Pi_{z}^{\left(n^{\prime}\right)},
$$

where $\Pi_{z}$ is the total power radiated from the baffle plane into the body. Having $\Pi_{z}^{(n)}$, it is easy to guess the absolute value of the Poynting vector $\Pi^{(n)}$ for given Bloch order by taking into account the wave propagation direction described by its wave-vector $\left(\xi_{n}, \eta_{n}\right)$ :

$$
\Pi^{(n)}=\frac{k}{\eta_{n}} \Pi_{z}^{(n)}, \quad a\left(\theta_{n}\right)=\sqrt{2 \Pi^{(n)}},
$$

$\eta_{n}=\sqrt{k^{2}-\xi_{n}^{2}}$ and $\theta_{n}=\operatorname{acos}\left(\left(\eta_{n} / k\right)\right.$. The radiation pattern $a(\theta)$ evaluated this way exploits the results already obtained in evaluation of the acoustic field at the baffle plane.

Noticing that $v=0$ outside the domain of given constant pressure $p_{l}=p_{0}$ (the current discussion concerns the pressure in only one slot, $l=0$; the generalization is straightforward), the delivered power is:

$$
P=\operatorname{Re}\left\{v p^{*}\right\} / 2=\operatorname{Re} \int_{-w / 2}^{w / 2} p_{l}^{*} v(x) \mathrm{d} x / 2 \Rightarrow p_{l}^{*} \int_{-\Lambda / 2}^{\Lambda / 2} v(x) \mathrm{d} x / 2 .
$$

The last integral can be evaluated using the Dougall identity (ERDELY et al., 1953) which can be derived from Eq. (16) by applying identities mentioned below Eq. (15):

$$
\frac{1}{2} \int_{-\Lambda / 2}^{\Lambda / 2} v(x) \mathrm{d} x=g_{\infty} \frac{\pi}{K} \sum_{m} \alpha_{m} P_{-m-r / K}(-\cos \Delta) .
$$

One can check by computations that the evaluated delivered $(P)$ and outgoing $\left(\Pi_{z}\right)$ acoustic powers agree nicely (up to six digits; similar accuracy takes place in the scattering problem discussed further below).

In the numerical examples presented in Fig. 2 both the pressure on the baffle plane $p(x)$ and the radiation pattern $|p(\theta)|$ are presented, computed for several values of slot width $w$ and for the baffle period $\Lambda=0.7 \lambda$, Figs. 2a,b, and $\Lambda=\lambda$, 
Figs. 2c,d. Note that the pressure built-up on baffles can significantly modify the radiation pattern and it is even able to extinguish the radiation at certain directions $\theta$. In the above examples we applied the given pressure in one slot only: $p_{l}=\delta_{l 0}$. In Fig. 3 the radiation pattern is computed for the case of 5 active slots excited with the chosen phase shift $p_{l}=\exp (j k \Lambda \sin \theta)$, for $l=-2, \ldots, 2$ and $p_{l}=0$ otherwise. The steering angle is chosen $\theta=30^{\circ}$ and $0^{\circ}$ for comparison. The steering angle differs slightly from the applied value $\theta=30^{\circ}$. This difference can be diminished by taking more active slots. At the same time the width of the main lobe gets smaller and the side lobe level remains unchanged. In Fig. $3 \mathrm{~b}$ the corresponding distribution of the pressure field on the baffle plane is presented. The grating lobe which appears for the steering angle $\theta=30^{\circ}$ is due to the spatial sampling theorem $(\Lambda \leq \lambda / 2)$ violation in the considered case of $\Lambda=0.7 \lambda$. This is further discussed in the next section (see Fig. 5, Eq. 30 and the discussion therein after). For convenience, the real and imaginary parts of the pressure distribution in Figs. 2, 3 are shown for positive and negative values of $x$-coordinate respectively, since in the above examples the odd number of active slots is considered, resulting in the symmetry of the graph of $p(x)$ with respect to the origin.

$\Lambda / \lambda=0.7:-w / \lambda=0.6$

a)

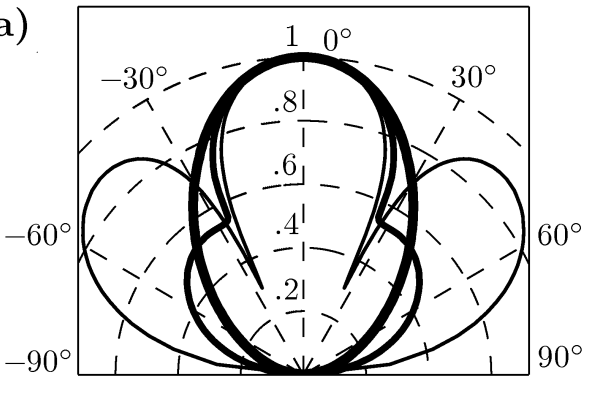

$w / \lambda=0.3 ;-w / \lambda=0.1$
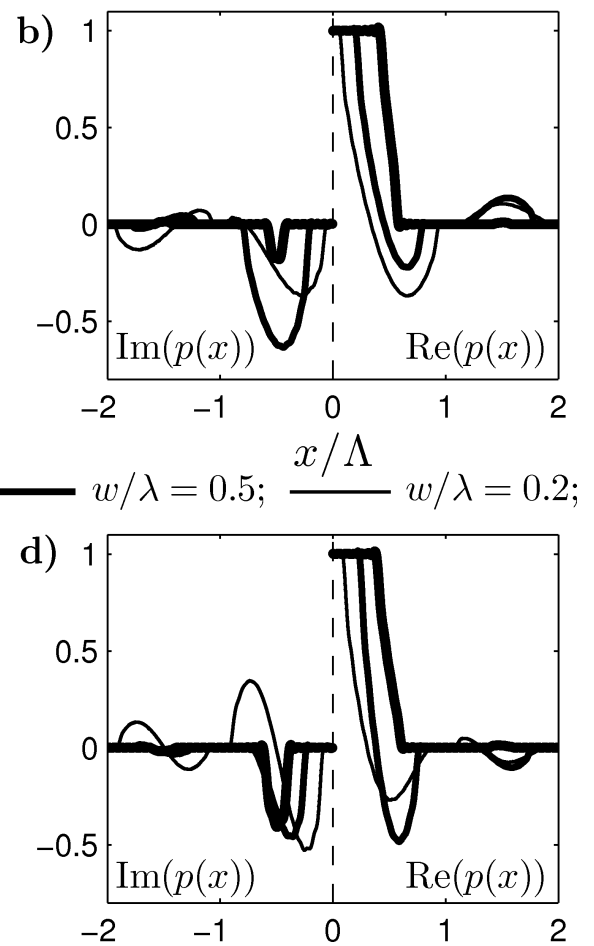

Fig. 2. Radiation pattern $|p(\theta)|$ and pressure distribution on the baffle plane $p(x)$ for $\Lambda / \lambda=0.7,(\mathrm{a}),(\mathrm{b})$, and $\Lambda / \lambda=1,(\mathrm{c}),(\mathrm{d})$ and different slot width $w$. 

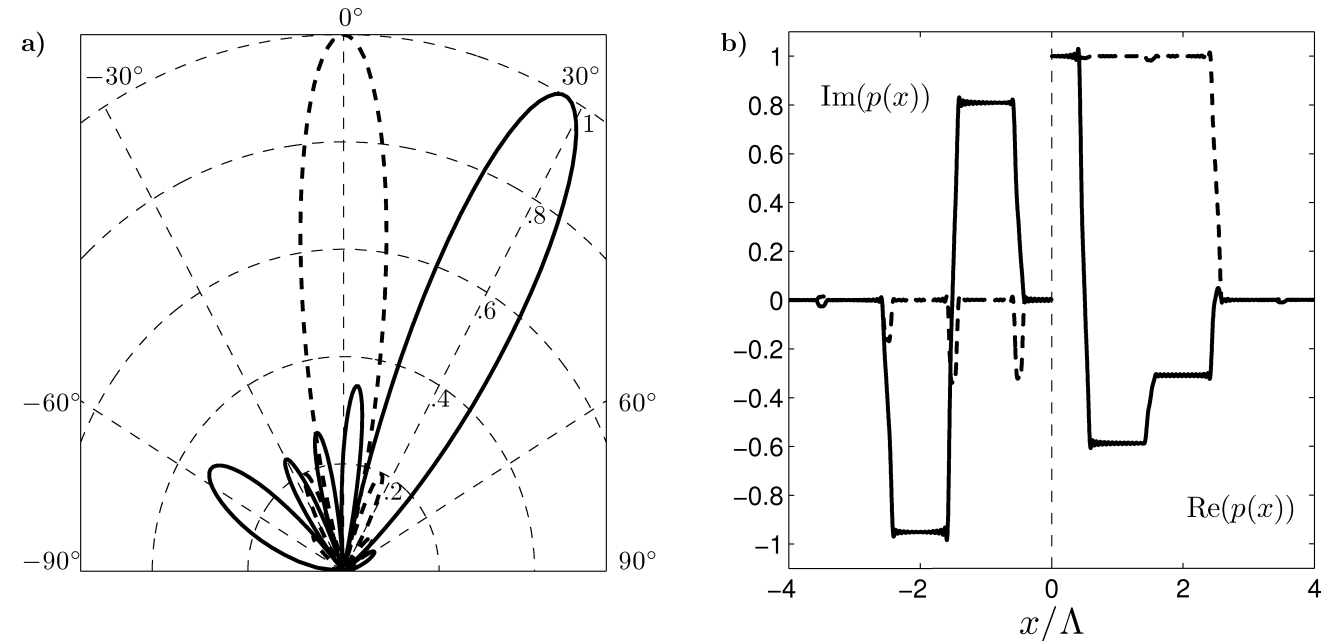

Fig. 3. Radiation pattern $|p(\theta)|$ for $\Lambda / \lambda=0.7, w / \lambda=0.6$ and 5 active slots excited with linear phase shift and steering angle $\theta=30^{\circ}$ (solid line) and $0^{\circ}$ (dashed line).

\subsection{A receiver beam-forming - scattered sound pressure on baffles}

The total force exerted by the incident wave on a baffle is (assuming $p(x)=0$ between baffles):

$$
\bar{p}=\int_{w / 2}^{\Lambda-w / 2} p(x) \mathrm{d} x=\int_{0}^{\Lambda} \sum_{n} p_{n} e^{-j r_{n} x} \mathrm{~d} x .
$$

Figure 4a presents Bragg orders of the scattered wave-field for the case of small value of $K / k$. After simple evaluation of integrals where $p_{n}=\int q_{n} d x$ evaluated from Eq. (10), one obtains:

$$
\bar{p}=2 j \sin (r \Lambda / 2) e^{-j r \Lambda / 2} \sum_{m} \alpha_{m} \sum_{n} \frac{S_{n-m} P_{n-m}}{(r+n K)^{2}},
$$

which series converges fast and can be easily evaluated numerically. The directional characteristics of the wave detection by baffles for several values of slot width $w$ and the baffle period $\Lambda=0.5 \lambda$ and $\Lambda=0.7 \lambda$ are shown in Fig. $4 \mathrm{~b}$ and $\mathrm{c}$ respectively. In Fig. $4 \mathrm{~d}$ the real and imaginary parts of $\bar{p}(\theta)$ are shown for $w=0.6 \lambda, w=0.3 \lambda$ and $\Lambda=0.7 \lambda$. The inflection point of the curves is clear. The phenomenon takes place when the -1st Bragg component of the scattered field approaches the tangential direction of propagation with respect to the baffle system, that is at $r-K \rightarrow-k$. In the above examples, the value $r=10^{-4} k$ was used instead of $r=0$ in order to avoid evaluation of the corresponding limits $r \rightarrow 0$ (DANICKI, 1999). 

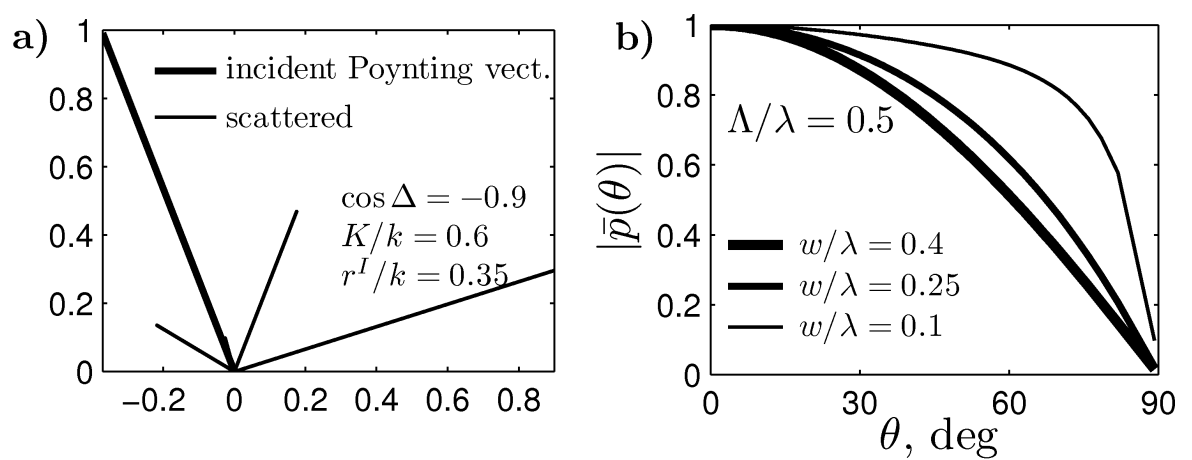

$\Lambda / \lambda=0.7$
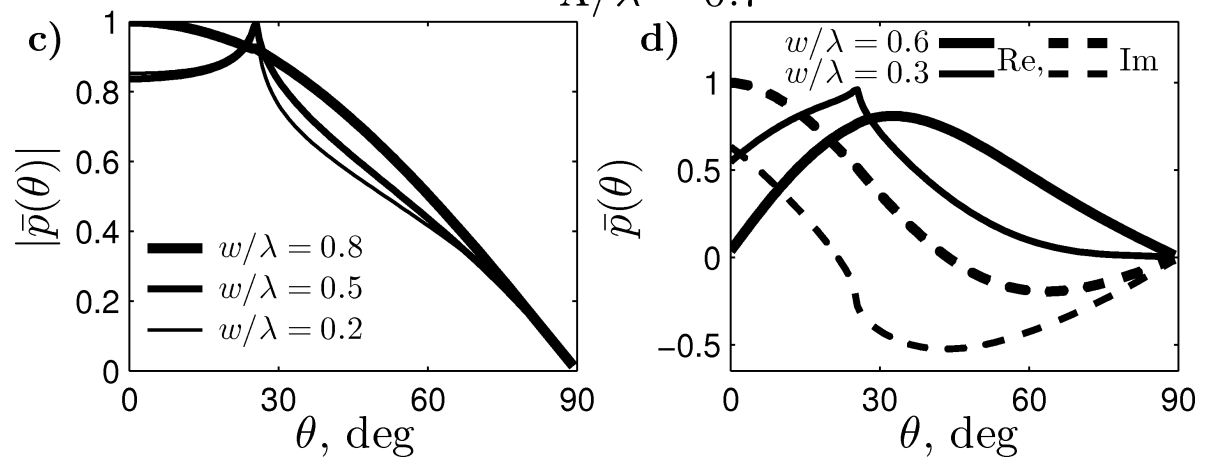

Fig. 4. Directional characteristic of the wave detection by baffles $\bar{p}(\theta)$ for different $\Lambda$ and several $w$.

In the example of receiver wave-beam synthesis, the output signal is the Hamming-windowed (CoOK, BeRnFELD, 1967) sum of signals $\bar{p}_{l}$ from 20 transducer elements:

$$
\begin{gathered}
S(\theta)=\sum_{n=1}^{20} \bar{p}_{n} W_{n} e^{j x_{n} k \cos \vartheta}, \quad x_{n}=(n-10.5) \Lambda, \\
W_{n}=0.08+0.92 \cos ^{2}(\pi(n-10.5) / 20),
\end{gathered}
$$

where $\vartheta$ is the chosen observation direction: $0^{\circ}$ or $30^{\circ}$ in the examples presented in Fig. 5 , while the angle of incidence $\theta$ sweeps over an entire domain $\left(\bar{p}_{n}\right.$ depends on $r=k \sin \theta$ ). The results are compared with ideal cases of point receivers responding to the incident wave by $\bar{p}_{n}=\exp \left(-j r x_{n}\right)$.

Since the pitch $\Lambda=0.7 \lambda$ does not obey the spatial sampling theorem $(\Lambda \geq$ $\lambda / 2$ ), the grating lobes appear for $\vartheta=30^{\circ}$ in Fig. 5b. The exact formula

$$
\theta_{m}=\arcsin \{ \pm m \lambda / \Lambda-\sin \vartheta\}
$$

yields for $\vartheta=30^{\circ}$ and $m=-1$ the value $\theta_{-1}=-68^{\circ}$ which is in a good agreement with numerical results in Fig. 5b. 


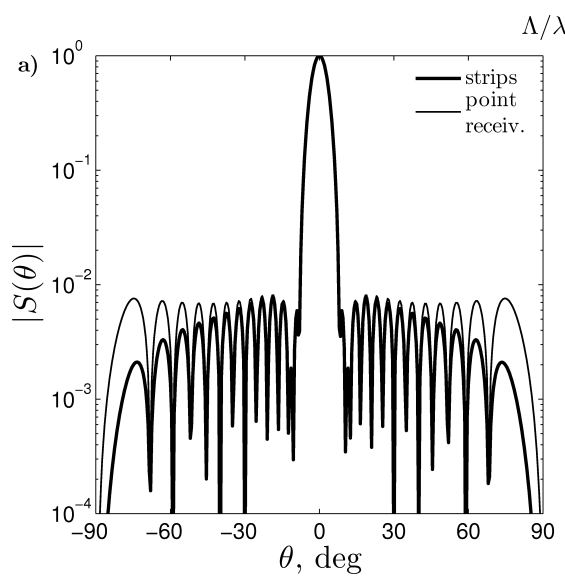

$\Lambda / \lambda=0.7, w / \lambda=0.6$

Fig. 5. Directional characteristic of the wave detection by baffles computed from Eq. (29) for observation angles: a) $\vartheta=0^{\circ}$ and b) $\vartheta=30^{\circ}$; $\Lambda=0.7 \lambda, w=0.6 \lambda$ in both cases.

\section{Conclusions}

The presented examples confirm that the applied method is worth consideration for numerical experiments concerning the beam-forming systems. It is very efficient numerically, yielding all interesting characteristics of the system within the same simple analysis, and yields results satisfying the energy conservation law, the valuable feature allowing us to check the computed results easily. This is important for evaluation of non-stationary signals comprising wide spectrum, where computations must be repeated for many frequencies.

Further modification of the presented method makes it applicable for analyzing finite transducer array, periodic or having different transducer elements with different width and spacing (DANicki, 2004; TASINKEvyCH, 2004). Such modification may help reducing spurious effects connected with abrupt ends of the transducer system, which is considered as quite difficult for analysis. Authors' intention is to investigate this problem in future works. The proposed method seems to be particularly useful and suitable for modeling receiving transducer array in the system where the observation wave-beam is sweeping over wide range of angles.

\section{Acknowledgment}

One of the authors (YT) acknowledges the support by the Ministry of Research and Higher Education under Grants No. N-N 518-3821-37.

\section{References}

1. Ahmad R., Kundu T., Placko D. (2005), Modeling of phased array transducers, Journal of the Acoustical Society of America, 117, 4, 1762-1776. 
2. BlotekJa K., Ingebrig K.A., Skeie H. (1973), Methods for analyzing waves in structures consisting of metal strips on dispersive media, IEEE Transactions on Electron Devices, ED20, 12, 1133-1138.

3. Boersma J., DANICKI E. (1993), On the solution of an integral equation arising in potential problems for circular and elliptic disks, SIAM Journal on Applied Mathematics, 53, 4, 931-941.

4. Chino R.Y., Thomas L.J. (1994), Analytic evaluation of sampled aperture ultrasonic imaging techniques for NDE, IEEE Transactions on ultrasonics ferroelectrics and frequency control, 41, 4, 484-493.

5. Cook C., Bernfeld M. (1967), Radar Signals, An Introduction to Theory and Application, Chap. 7, Academic Press, New York.

6. Crombie P., Bascom P.A.J., Cobbold R.S.C. (1997), Calculating the pulsed response of linear arrays: Accuracy versus computational efficiency, IEEE Transactions on Ultrasonics Ferroelectrics and Frequency Control, 44, 5, 997-1009.

7. DANicki E. (1996), Strip electrostatics - Spectral approach, 1996 IEEE Ultrasonics Symposium, Proceedings, 193-196.

8. DANICKI E.J. (1999), Resonant phenomena in bulk-wave scattering by in-plane periodic cracks, Journal of the Acoustical Society of America, 105, 1, 84-92.

9. DAnicki E.J. (2002), Scattering by periodic cracks and theory of comb transducers, Wave Motion, 35, 4, 355-370.

10. DAnicki E.J. (2004), Electrostatics of interdigital transducers, IEEE Transactions on Ultrasonics Ferroelectrics and Frequency Control, 51, 4, 444-452.

11. Drinkwater B.W., Wilcox P.D. (2006), Ultrasonic arrays for non-destructive evaluation: A review, NDT \& E International, 39, 7, 525-541.

12. Erbas B., Abrahams I.D. (2007), Scattering of sound waves by an infinite grating composed of rigid plates, Wave Motion, 44, 4, 282-303.

13. Erdelyi A., Magnus W., Oberhettinger F., Tricomi F.G. (1953), Higher Transcendental Functions, vol. 1, Chap. 3, McGraw-Hill, New York, 166-167.

14. Fedoryuk M.W. (1997), The Steepest Descent Method, Chap. 3, Nauka, Moscow.

15. Felsen L.B., Marcuvitz N. (1973), Radiation and Scattering of Waves, vol. 1, Chap. 4, Prentice Hall, Englewood Cliffs.

16. Jensen J.A., Svendsen N.B. (1992), Calculation of pressure fields from arbitrary shaped, apodized, and excited ultrasound transducers, IEEE Transactions on Ultrasonics Ferroelectrics and Frequency Control, 39, 2, 262-267.

17. Kramb V.A. (2007), Use of phased array ultrasonics for automated aerospace testing applications, Materials Evaluation, 65, 1, 67-73.

18. Kuhnicke E. (2007), Plane arrays - Fundamental investigations for correct steering by means of sound field calculations, Wave Motion, 44, 4, 248-261.

19. Lerch R. (1990), Simulation of piezoelectric devices by 2-dimensional and 3-dimensional finite-elements, IEEE Transactions on Ultrasonics Ferroelectrics and Frequency Control, 37, 3, 233-247. 
20. Lingvall F., Olofsson T., Stepinski T. (2003), Synthetic aperture imaging using sources with finite aperture: Deconvolution of the spatial impulse response, Journal of the Acoustical Society of America, 114, 1, 225-234.

21. McNab A., Cochran A., Campbell M.A. (1990), The calculation of acoustic fields in solids for transient normal surface force sources of arbitrary geometry and apodization, Journal of the Acoustical Society of America, 87, 4, 1455-1465.

22. Selfridge A.R., Kino G.S., Khuriyakub B.T. (1980), A theory for the radiation pattern of a narrow-strip acoustic transducer, Applied Physics Letters, 37, 1, 35-36.

23. Seo C.H., Yen J.T. (2007), 256x256 2-D array transducer with row-column addressing for 3-D imaging, 2007 IEEE Ultrasonics Symposium, Proceedings, 2381-2384.

24. TASinkeVych Y. (2004), Methods of IDT charge spatial spectrum evaluation, Journal of Technical Physics, 45, 155-172.

25. Thomenius K.E. (1996), Evolution of ultrasound beamformers, 1996 IEEE Ultrasonics Symposium, Proceedings, 1615-1622.

26. Trots I., Nowicki A., Lewandowski M. (2008), Laboratory setup for synthetic aperture ultrasound imaging, Archives of Acoustics, 33, 4, 573-580.

27. Trots I., Nowicki A., Lewandowski M. (2009), Synthetic transmit aperture in ultrasound imaging, Archives of Acoustics, 34, 4, 685-695.

28. Wоон S.C., Sнi Y.J. (1998), Influence of phased array element size on beam steering behavior, Ultrasonics, 36, 6, 737-749.

29. Woon S.C., Shi Y.J. (1999), Three-dimensional beam directivity of phase-steered ultrasound, Journal of the Acoustical Society of America, 105, 6, 3275-3282.

30. Yaralioglu C.G., Ergun A.S., Khuri-Yakub B.T. (2005), Finite-element analysis of capacitive micromachined ultrasonic transducers, IEEE Transactions on Ultrasonics Ferroelectrics and Frequency Control, 52, 12, 2185-2198.

31. Yen J.T., Steinberg J.P., Smith S.W. (2000), Sparse 2-D array design for real time rectilinear volumetric imaging, IEEE Transactions on Ultrasonics Ferroelectrics and Frequency Control, 47, 1, 93-110.

32. Zemp R.J., AbBey C.K., Insana M.F. (2003), Linear system models for ultrasonic imaging: application to signal statistics, IEEE Transactions on Ultrasonics Ferroelectrics and Frequency Control, 50, 6, 642-654. 